\title{
Purely elastic instabilities in a microfluidic flow focusing device
}

\author{
P. Ballesta and M. A. Alves* \\ Faculdade de Engenharia da Universidade do Porto, CEFT, Dep. Engenharia Química, \\ Rua Dr. Roberto Frias, 4200-465 Porto, Portugal
}

(Received 8 August 2016; published 4 May 2017)

\begin{abstract}
In this work, we investigate the behavior of dilute and semidilute polymer solutions flowing in a microfluidic flow focusing device, in which an inlet stream is stretched by two balanced lateral streams. By varying the flow rates in the inlet and lateral channels and their ratio, several types of flow transitions are observed and the resulting velocity fields are analyzed both upstream and downstream of the intersection. At high flow rates, the flow becomes chaotic and the path to this state depends on both the Weissenberg number and the imposed Hencky strain. The thresholds of successive elastic instabilities leading to the chaotic state are also investigated using time-resolved microparticle imaging velocimetry.
\end{abstract}

DOI: 10.1103/PhysRevFluids.2.053301

\section{INTRODUCTION}

Understanding the flow of complex fluids through a cross-shaped channel is important for both fundamental research and practical applications. Cross-shaped channels are commonly used to investigate the flow of viscoelastic fluids in microfluidic devices where inertial effects are usually negligible. The standard cross geometry consists of four orthogonal channels with independent flow rates and can be used in a variety of configurations. The most common configuration is the cross-slot extensional flow which was extensively studied for polymeric solutions both experimentally [1-3] and numerically [4]. In this configuration, the flow rates are opposite in opposing channels, with two balanced inlet flow rates, and two balanced outlet flow rates, creating a stagnation point at the center of the intersection and generating a strong extensional flow in the central region, with relevant applications in extensional rheometry [5-7] and the study of the deformation of biological samples [8-10]. Recently, flow focusing devices have also received particular attention [11-14]. In this configuration, three channels are used to deliver fluids, and the flow from the central channel is focused between balanced sheath streams coming in from the lateral channels. This can be used to create calibrated droplets [11] and study elastic instabilities at high extensional rates [12,15]. Both microfluidic cross-slot and flow focusing devices $[3,11,13]$ combine small length scales and strong extensional flow fields which enable us to investigate elastic instabilities and eventually elastic turbulence under negligible inertia [16-20].

In this experimental work, we investigate the onset of purely elastic flow instabilities of polymeric solutions in a microfluidic flow focusing device using flow visualization and micro-particle image velocimetry (PIV). The advantage of this configuration over other geometries known to trigger elastic turbulence [21-23] is the ability to independently vary the lateral and the central flow rates hence changing the extensional strain rate independently of the strain imposed to the fluid injected through the central inlet. Previous works have been done on this flow configuration either numerically [12] or experimentally [13], and in both cases a similar behavior was found: at low flow rates the flow is steady and symmetric, and above a critical flow rate an elastic instability renders the flow asymmetric

\footnotetext{
*mmalves@fe.up.pt
}

Published by the American Physical Society under the terms of the Creative Commons Attribution 3.0 License. Further distribution of this work must maintain attribution to the author(s) and the published article's title, journal citation, and DOI. 

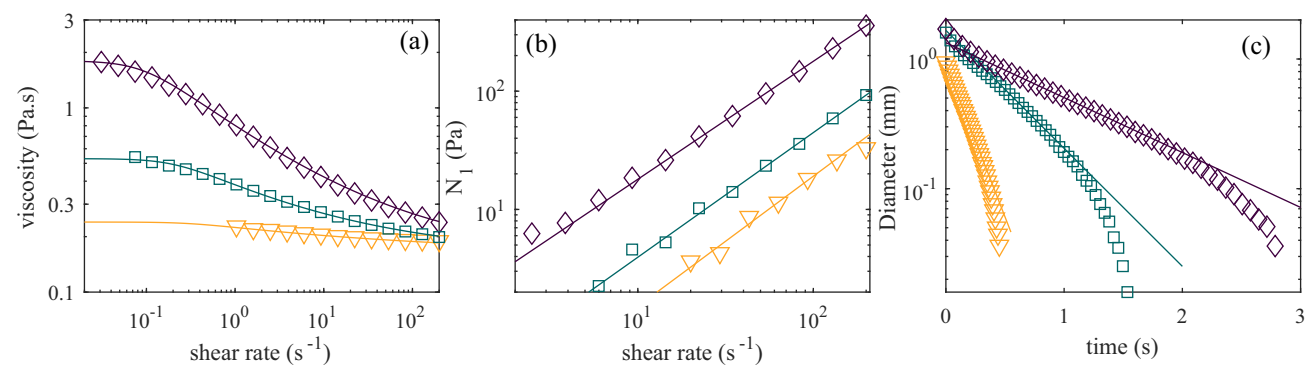

FIG. 1. (a) Shear viscosity $\eta$ as a function of the shear rate $\dot{\gamma}$ measured at $T=22^{\circ} \mathrm{C}$, for PAA polymer solutions at $c=300 \mathrm{ppm}$ (diamonds), $c=150 \mathrm{ppm}$ (squares), and $50 \mathrm{ppm}$ (triangles). The continuous lines are fits using the Carreau model for shear viscosity: $\eta_{\infty}=0.16 \mathrm{~Pa} \cdot \mathrm{s}, \eta_{0}=1.80 \mathrm{~Pa} \cdot \mathrm{s}, \tau_{e}=11.0 \mathrm{~s}$, and $m=0.61$ for $c=300 \mathrm{ppm} ; \eta_{\infty}=0.16 \mathrm{~Pa} \cdot \mathrm{s}, \eta_{0}=0.53 \mathrm{~Pa} \cdot \mathrm{s}, \tau_{e}=4.8 \mathrm{~s}$, and $m=0.68$ for $c=150 \mathrm{ppm}$; and $\eta_{\infty}=0.16 \mathrm{~Pa} \cdot \mathrm{s}, \eta_{0}=0.24 \mathrm{~Pa} \cdot \mathrm{s}, \tau_{e}=3.2 \mathrm{~s}$, and $m=0.82$ for $c=50 \mathrm{ppm}$. (b) First normal stress difference $N_{1}$ as function of shear rate $\dot{\gamma}$ for the same fluids. The continuous lines are fits by a power law function $N_{1}=\alpha \dot{\gamma}^{q}$, with $N_{1}$ in $\mathrm{Pa}$ and $\dot{\gamma}$ in $\mathrm{s}^{-1}: \alpha=1.81$ and $q=0.998$ for $c=300 \mathrm{ppm} ; \alpha=0.348$ and $q=1.05$ for $c=150$; and $\alpha=0.120$ and $q=1.10$ for $c=50$ ppm. (c) Diameter versus time measured in the CaBER ${ }^{\mathrm{TM}}$ 1 rheometer at $T=22{ }^{\circ} \mathrm{C}$, where the continuous lines are the exponential fits of the filament diameters as function of time in the elasto-capillary regime, from which $\lambda$ is determined.

but still steady; at higher flow rates, the velocity field becomes time dependent. Oliveira et al. [12] argued that the transition to asymmetric flow is a stress-relief mechanism, and a review on these instabilities can be found in Ref. [24]. In a recent work in a millifluidic T-junction device, Varshney et al. [15] observed a direct transition to unsteady flow, without the onset of a steady asymmetric flow. As will be shown, these two flow instabilities may occur in the setup used in this work, depending on the velocity ratio and the inlet flow rate in the main channel, eventually resulting in chaotic flows at even higher flow rates, a regime not previously investigated in detail in flow focusing devices, and which is addressed in this work. The remaining of this paper is structured as follows: we start by presenting the rheology of the fluids and the experimental setup, followed by a description of the experimental results. The paper ends with a discussion of the results and how the flow field changes with the relevant dimensionless parameters.

\section{POLYMERIC SOLUTIONS}

The fluids studied are dilute and semidilute solutions of polyacrylamide (PAA), with an average molecular weight $M=18 \times 10^{6} \mathrm{Da}$ (Polysciences), in a mixture of glycerol and water with 88.7 $\pm 0.2 \%$ per weight of glycerol. The PAA concentrations tested were $c=300,150$, and $50 \mathrm{ppm}$ in weight, which correspond to $c / c^{*}=1.8,0.9$, and 0.3 respectively, where $c^{*} \simeq 170 \mathrm{ppm}$ is the estimated overlap concentration [3]. A small amount (400 ppm in weight) of spherical fluorescent tracer particles (diameter $\varnothing=1 \mu \mathrm{m}$, Nile red particles, Invitrogen) are added to measure the velocity field using micro-PIV (Dynamic Studio v3.41, Dantec Dynamics). To reduce the adhesion of tracer particles at the walls of the channels, sodium dodecyl sulfate (SDS) was added at a weight concentration of $110 \mathrm{ppm}$. Rheology measurements (shear viscosity $\eta$ and first normal-stress difference $N_{1}$ as a function of shear rate $\dot{\gamma}$ ) in steady shear flow were measured with a rotational rheometer (Physica MCR301, Anton Paar) with a cone-plate geometry of diameter $50 \mathrm{~mm}$ and angle $2^{\circ}$, using the final solutions with SDS and tracer particles. The resulting viscosity curves are similar to the rheology without tracers and SDS for the same concentrations of polymer. These fluids are viscoelastic and only slightly shear thinning as shown in Fig. 1, with the dilute solutions approaching a Boger fluid behavior [25]. Contrary to similar solutions in which salt was added [13,20,26,27], the shear thinning property of the solutions used in this work is relevant for the semidilute solution; the shear viscosity decreases roughly by a factor of four over the 

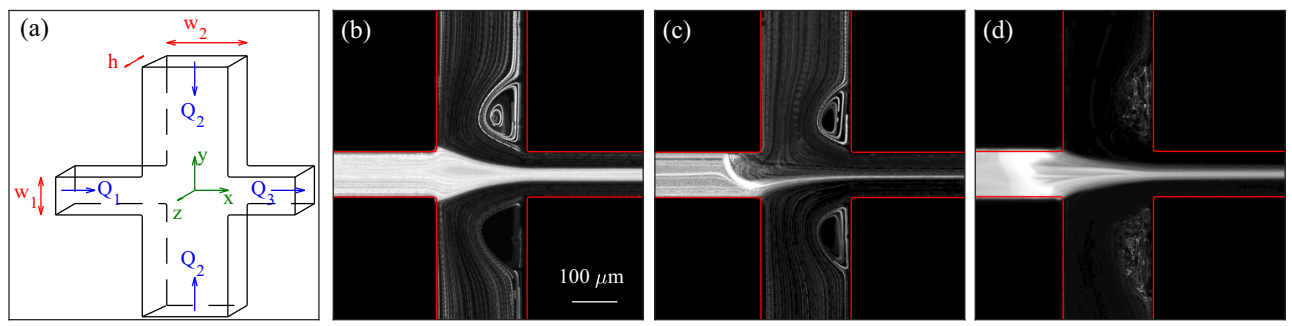

FIG. 2. (a) Schematic of the flow focusing device. (b-d) Images taken for long exposure times with rhodamine-tinted solutions in the central channel. The continuous (red) lines were added to highlight the location of the walls of the flow focusing device. Illustrative flow patterns for (b) stable flow (Wi $=0.30, \epsilon_{H}=1.10$ ), (c) asymmetric stable flow (Wi $\left.=1.4, \epsilon_{H}=2.94\right)$, and (d) time-dependent flow $\left(\mathrm{Wi}=7.3, \epsilon_{H}=3.04\right)$.

range of shear rates investigated. The steady shear viscosity curves were fitted with a Carreau model [28], $\eta=\eta_{\infty}+\left(\eta_{0}-\eta_{\infty}\right)\left[1+\left(\tau_{e} \dot{\gamma}\right)^{2}\right]^{(m-1) / 2}$, and the best fitting parameters are given in the caption of Fig. 1(a). The extensional relaxation times, $\lambda$, were measured in extensional flow using a capillary breakup extensional rheometer (CaBER, Haake $\mathrm{CaBER}^{\mathrm{TM}} 1$, Thermo Electron), and the following average values were obtained: $\lambda=0.34 \mathrm{~s}$ at $c=300 \mathrm{ppm}, \lambda=0.16 \mathrm{~s}$ at $c=150$ ppm, and $\lambda=0.062 \mathrm{~s}$ at $c=50 \mathrm{ppm}$ [see Fig. 1(c)]. The relaxation times vary nearly linearly with polymer concentration for the fluids tested, in agreement with the measurements of Sousa et al. [29] for aqueous PAA solutions.

\section{EXPERIMENTAL SET-UP}

The microchannels are made in house in polydimethylsiloxane (PDMS), with custom SU-8 molds. We checked, through bright light imaging on different microchannels produced independently on different days, that the dimensions were constant within $\pm 2 \mu \mathrm{m}$. Moreover, due to the elasticity of the channels, we observed a deformation of the channels of about 5\% in the $x y$ plane for the higher flow rates, well above the onset of time-dependent flow. The geometry can be seen as two straight channels crossing, as illustrated in Fig. 2(a). The first channel forms the central entry, and the exit and has a width $w_{1}=100 \mu \mathrm{m}$. The second channel is orthogonal to the first and composes the lateral entrances with a constant width $w_{2}=200 \mu \mathrm{m}$. The depth of the channels is constant $h=110 \mu \mathrm{m}$. The corners of the intersection are rounded with a radius of $5 \mu \mathrm{m}$. Both lateral branches have the same length $(5 \mathrm{~mm})$ from inlet to cross section, the inlet channel is $10 \mathrm{~mm}$ long, while the outlet channel is longer $(30 \mathrm{~mm})$ to measure the evolution of the velocity field as the fluid flows away from the intersection.

Constant flow rates in the channels are imposed using Hamilton syringes on a low-pressure neMESYS syringe pump (Cetoni $\mathrm{GmbH}$ ). To minimize the oscillations in the three imposed inlet flow rates, induced by the discrete microsteps of the motor of the syringe pump, the volume of the syringes was chosen to ensure that the piston velocity is at least one order of magnitude higher than the pulsation free rate given by the manufacturer. The outlet channel is open to the atmosphere. The entry flow rate is $Q_{1}$, the two side flow rates are equal and noted $Q_{2}$, while the exit flow rate is $Q_{3}=Q_{1}+2 Q_{2}$. The average velocities are $U_{1}=Q_{1} /\left(h w_{1}\right), U_{2}=Q_{2} /\left(h w_{2}\right)$, and $U_{3}=Q_{3} /\left(h w_{1}\right)$, respectively.

A flow focusing experiment can be characterized by two parameters: the degree of deformation of the fluid in the central channel and how fast this deformation occurs. Different dimensionless numbers can be used to describe the instabilities [30,31]. The degree of deformation is generally described by the Hencky strain, $\epsilon_{H}=\ln \left(U_{3} / U_{1}\right)$. In cases where the normal stresses generated by the shear flow is the main cause of instability [17,20], we can define a shear Weissenberg number, $\mathrm{Wi}_{\text {shear }}=N_{1} /\left(2 \sigma_{p}\right)$, where $N_{1}$ and $\sigma_{p}$ are the first-normal stress difference and polymeric component of the shear stress evaluated at the wall conditions. Another dimensionless number one can consider 
is the Deborah number De, which is defined as the ratio between the relaxation time of the fluid and a characteristic time scale of the flow, De $=\lambda / t_{f}$. If we estimate $t_{f}$ as the time needed for a fluid element to accelerate through the intersection from $U_{1}$ at $x \simeq-w_{2} / 2$ to $U_{3}$ at $x \simeq w_{2} / 2$, assuming a linear increase of the velocity in the extensional flow, we obtain De $=\lambda\left(U_{3}-U_{1}\right) /\left[w_{2} \ln \left(U_{3} / U_{1}\right)\right]$. We can also define a Weissenberg number dependent of the strain rate $\dot{\epsilon}, \mathrm{Wi}=\lambda \dot{\epsilon}$, which is important in strong extensional flows [5]. In our setup, these three values are related and can be used in combination with $\epsilon_{H}$ to describe the flow of a viscoelastic fluid. Here the accessible range of $\epsilon_{H}$ results in similar values for $\mathrm{Wi}, \mathrm{Wi}_{\text {shear }}$, and De. Therefore, in the following, we will use $\epsilon_{H}$ and Wi to describe the flow conditions. We have verified that this choice does not significantly change the results presented here. When calculating the Weissenberg number, the strain rate is estimated as $\dot{\epsilon}=\left(U_{3}-U_{1}\right) / w_{2}$, thus $\mathrm{Wi}=\lambda\left(U_{3}-U_{1}\right) / w_{2}$. Expressed as functions of the imposed flow rates, the Weissenberg number and the Hencky strain are Wi $=2 Q_{2} \lambda /\left(w_{1} w_{2} h\right)$ and $\epsilon_{H}=\ln \left(1+2 Q_{2} / Q_{1}\right)$, respectively. The flow rate in the exit channel ranges from $2.1 \mathrm{nl} / \mathrm{s}$ to $150 \mathrm{nl} / \mathrm{s}$, which correspond to estimated shear rates $\dot{\gamma} \simeq 2 U_{3} / w_{1}=2 Q_{3} /\left(h w_{1}^{2}\right)$ in the range $4-275 \mathrm{~s}^{-1}$. The lowest limit of the flow rate is imposed by the size of the smallest syringes available, while the high shear rate limit depends on the resistance of the channels. The resulting Reynolds number $\left(\operatorname{Re}=\rho U_{3} w_{1} / \eta\right)$ ranges approximately from $10^{-4}$ to 0.01 where $\eta$ is the shear viscosity measured at the characteristic shear rate $\dot{\gamma} \simeq 2 U_{3} / w_{1}$. Inertia is negligible at such low Reynolds number flows, therefore the instabilities observed are purely elastic.

The visualization setup includes a high-speed camera (Miro M340, Vision Research) and a double pulsed high-repetition rate laser (LDY301, Litron Lasers), which can be operated up to $10 \mathrm{kHz}$. The pulsed laser is connected to an inverted microscope (Olympus, IX83) using a liquid optical fiber and an adapter for collimating the light. We use an Olympus $10 \times$ objective with numerical aperture $\mathrm{NA}=0.3$ and a measurement depth of approximately $20 \mu \mathrm{m}$. The objective holder can be moved vertically with micrometric precision. We determine the location of the top and bottom walls of the channel by focusing at the immobile particles that adhere to the walls. Once the positions of the upper and lower walls are found, we place the focal plane of the objective at the middle. We chose to work at the midplane of the channel to minimize the effect of the focal depth of the objective because both the $z$ variation of the velocity profile and the flow component out of plane are expected to be less significant therein. Velocity fields are extracted from double frame images taken at a frequency $f_{a c q}$ at the middle plane of the channel with a time $\mathrm{d} t$ between two images in a pair. Movies are taken for a duration longer than $10 \lambda$ at a frame rate higher than $20 / \lambda$. At the same time $\mathrm{d} t$ is chosen to obtain a maximum displacement between a pair of images close to 5 pixels, or is fixed at $1 / f_{a c q}$ for the lowest applied flow rates. This methodology ensures at least one order of magnitude between the higher limit of the frequency window and the inverse of the relaxation time of the fluid and allows for a good resolution of the velocity field using the Adaptive Particle Image Velocimetry implemented in the Dynamic Studio software. All experiments were performed at room temperature, $T=22 \pm 1{ }^{\circ} \mathrm{C}$. This setup was first tested with Newtonian fluids (various glycerol-water mixtures) to estimate the noise in the measured velocity field induced by the syringe pump at various flow rates. Small amplitude oscillations were observed at the lower applied flow rates with the smaller syringes, which are consistent with the discrete nature of the pump stepper motor, but these oscillations disappear at higher flow rates such as those used in the viscoelastic flows reported here.

\section{RESULTS}

The velocity components along the central direction $x$ and the lateral direction $y$ are $u$ and $v$, respectively. Movies taken with a dyed fluid (rhodamine B, Sigma-Aldrich) at various Wi and $\epsilon_{H}$ are included as Supplementary Materials [32], and illustrative images of different flow regimes are presented in Figs. 2(b)-2(d). The solutions used in PIV experiments contain only fluorescent tracer particles and no dye. We also observed the appearance of large lip vortices at the reentrant corners of the downstream channel. These vortices are reminiscent of what is observed in contraction flow experiments [33]. Figure 2(d) illustrates the need of using fast velocimetry experiments to 

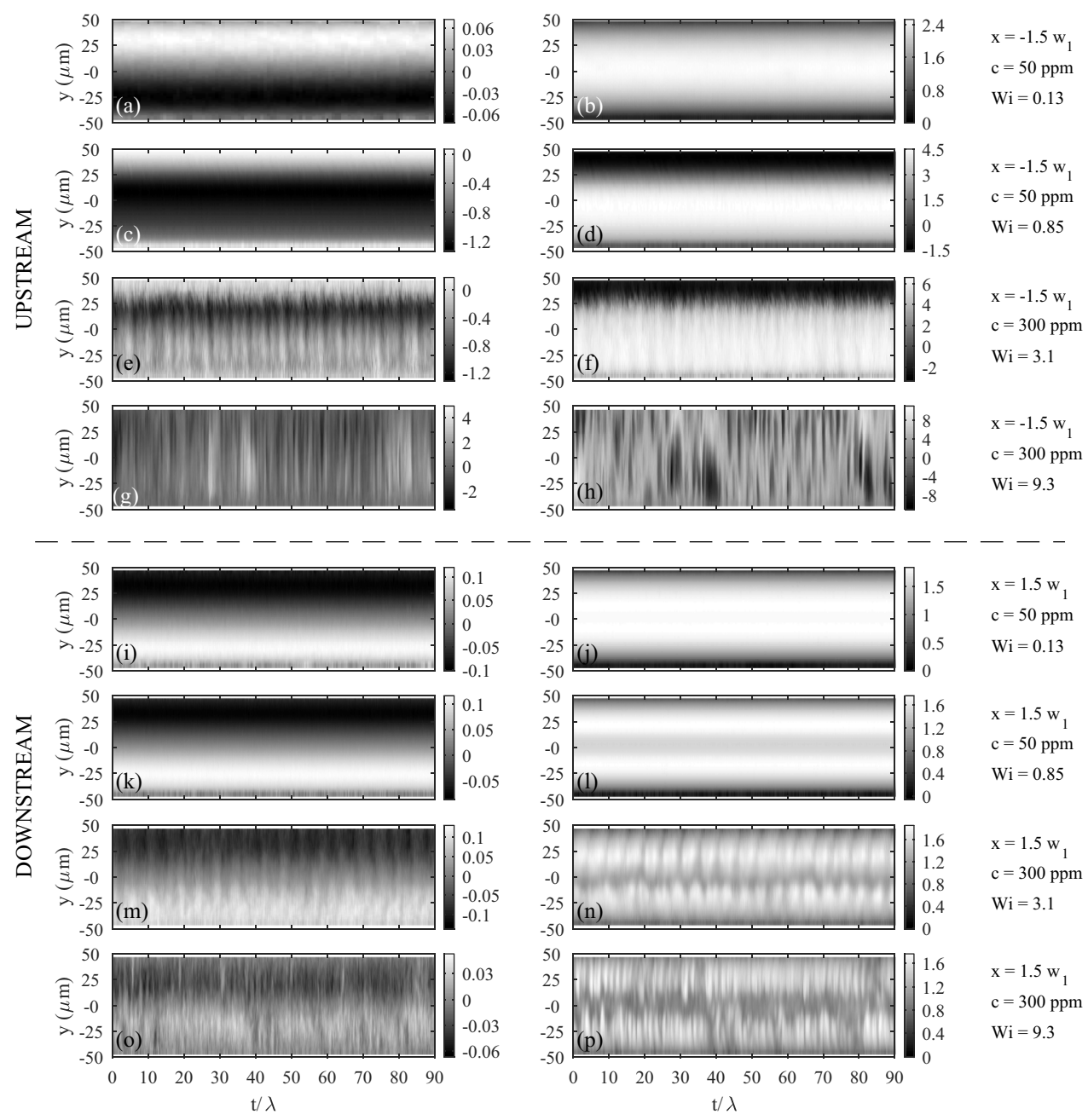

FIG. 3. Space-time diagrams of dimensionless velocity components (a, c, e, g) $v / U_{1}$, (b, d, f, h) $u / U_{1}$, (i, k, m, o) $v / U_{3}$, and $(\mathrm{j}, 1, \mathrm{n}, \mathrm{p}) u / U_{3}$ all taken for $\epsilon_{H}=3.04$.

obtain insight in the transient behavior of the downstream flow at high Wi. As can be seen in the entry channels, from the gray-scale intensity variation, the flow is complex, but in the exit channel the fluctuations have a higher wavelength. This clearly shows that the time scale of the velocity fluctuations is large compared to the time needed for a tracer to cross the visualization window.

\section{A. Transition to chaotic flow}

Figure 3 shows space-time diagrams of $u$ and $v$ at $x / w_{1}=-1.5$ (upstream) and $x / w_{1}=1.5$ (downstream) for a fixed $\epsilon_{H}$ and varying Wi. For the lowest Wi presented, the velocity field is steady and symmetric [Figs. 3(a) and 3(b)]. At higher Wi, the flow becomes asymmetric while remaining steady [Figs. 3(c) and 3(d)]. Increasing Wi, the velocity field becomes time dependent, with periodic oscillations [Figs. 3(e) and 3(f)]. As the flow rate is further increased, oscillations lose their long-time correlation, and the flow is described as semiperiodic. Finally, at high Wi, the flow becomes chaotic with large nonperiodic fluctuations of the velocity field [Figs. 3(g) and 3(h)]. This is similar to what was observed recently by Varshney et al. [15] in a T-channel, which can be seen as a flow 

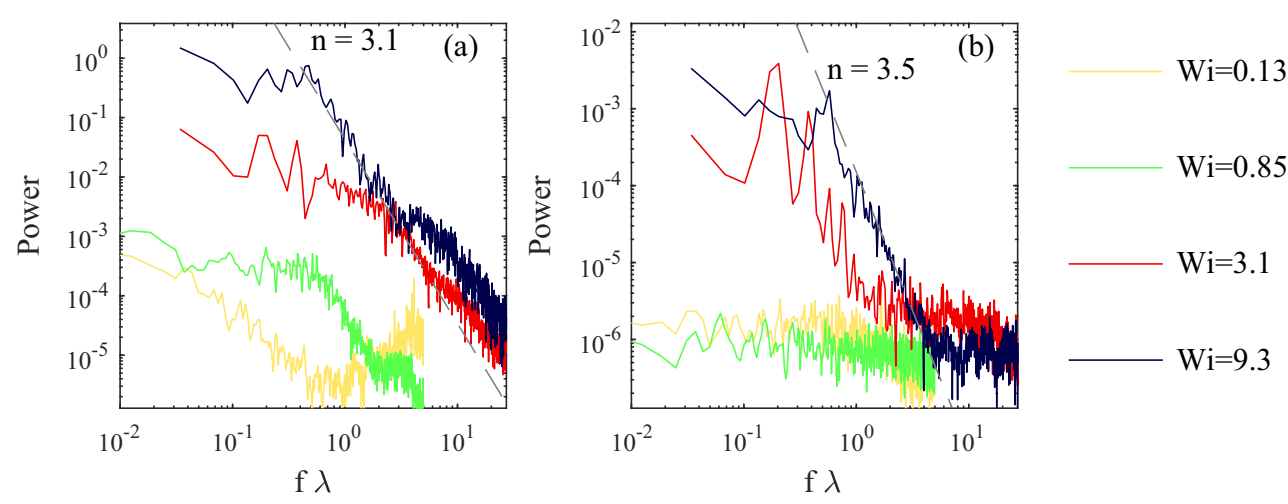

FIG. 4. (a) Power spectra of $u / U_{1}$ measured at $x=-1.5 w_{1}$ and $y=w_{1} / 4$ for $\epsilon_{H}=3.04$ as a function of the dimensionless frequency $f \lambda$ for $c=50 \mathrm{ppm}$ and $\mathrm{Wi}=0.13$ (very light orange), $c=50 \mathrm{ppm}$ and $\mathrm{Wi}=0.85$ (light green), $c=300 \mathrm{ppm}$ and $\mathrm{Wi}=3.1$ (red), and $c=300 \mathrm{ppm}$ and $\mathrm{Wi}=9.3$ (dark blue). (b) Power spectra of $u / U_{3}$ measured at $x=1.5 w_{1}$ for the same flows and fluids. (a, b) The dashed lines are a guide to the eye to illustrate typical power law decay in the elastic turbulence regime.

focusing device approaching an infinite $\epsilon_{H}$, although in the T-channel the steady asymmetric flow field was not observed. The fluctuations of the normalized velocities are relatively stronger upstream because of the smaller average velocity, and it is easier to portray in more detail the evolution of the velocity field upstream of the intersection. Experimentally, the fluctuations of $u$ and $v$ in the upstream region can be larger than $U_{1}$, while downstream $U_{3}$ is larger than the velocity fluctuations. However, the understanding of the dynamics of the velocity field as the fluid elements move away from the intersection is also important for the characterization of elastic effects, especially the decay of the temporal fluctuations downstream of the source of the perturbation. Figures 3(i)-3(p) present the space-time diagrams of the velocity profiles downstream of the intersection, at $x / w_{1}=1.5$. As can be seen, the flow fields upstream and downstream of the intersection are somehow different. Mainly, the upstream flow asymmetry does not affect significantly the downstream velocity field. Finally, $u / v$ is comparatively larger downstream of the intersection once instabilities set in, with $u / v \simeq 2$ upstream and $u / v \simeq 20$ downstream of the intersection.

Another way to look at the state of the flow is to compute the power spectrum $P$ of the velocity time series and measure the exponent of the power law decay with the frequency $f$ that occurs when a chaotic flow sets in, $P \propto f^{-n}$. We calculate the power spectrum by averaging power spectra obtained on smaller time windows, typically of 500 or 1000 time steps, with a Hann window. We have also computed the power spectra without any windowing and the results are similar. For Newtonian fluids, the power spectrum is nearly flat since the flow is steady, and mainly the noise of the velocity field measurements is observed in the power spectrum. On the other hand, in the elastic turbulence regime the power spectrum decays with $f$ as a power law with an exponent close to $n \simeq 3.5$ [21,34].

Figure 4 shows the power spectra of $u / U_{1}$ and $u / U_{3}$ presented in Fig. 3 both upstream [Fig. 4(a)] and downstream [Fig. 4(b)] of the intersection at $y=w_{1} / 4$. The power spectra measured downstream of the intersection [Fig. 4(b)] shows a transition from flat power spectra with low value of $P$, for the symmetric and asymmetric steady flows, to a power spectrum presenting marked peaks at a given frequency $(f \lambda \simeq 0.2)$ and its harmonics ( $\mathrm{Wi}=3.1)$, and finally a power law decay with an exponent $n \simeq 3.5$ at $\mathrm{Wi}=9.3$, which is consistent with previous studies on elastic turbulence $[21,26,34]$. We see that the transition from nearly flat power spectra to power law-like power spectra occurs alongside an increase of $P$, which reflects the increase of the amplitude and complexity of the fluctuations. However, power spectra of velocity measured upstream show a sharp decrease with increasing frequency, even when the velocity seems steady. This is due to the slow velocities upstream compared to the velocities downstream (here $Q_{1}=Q_{3} / 21$ ), and the limit of our set-up 

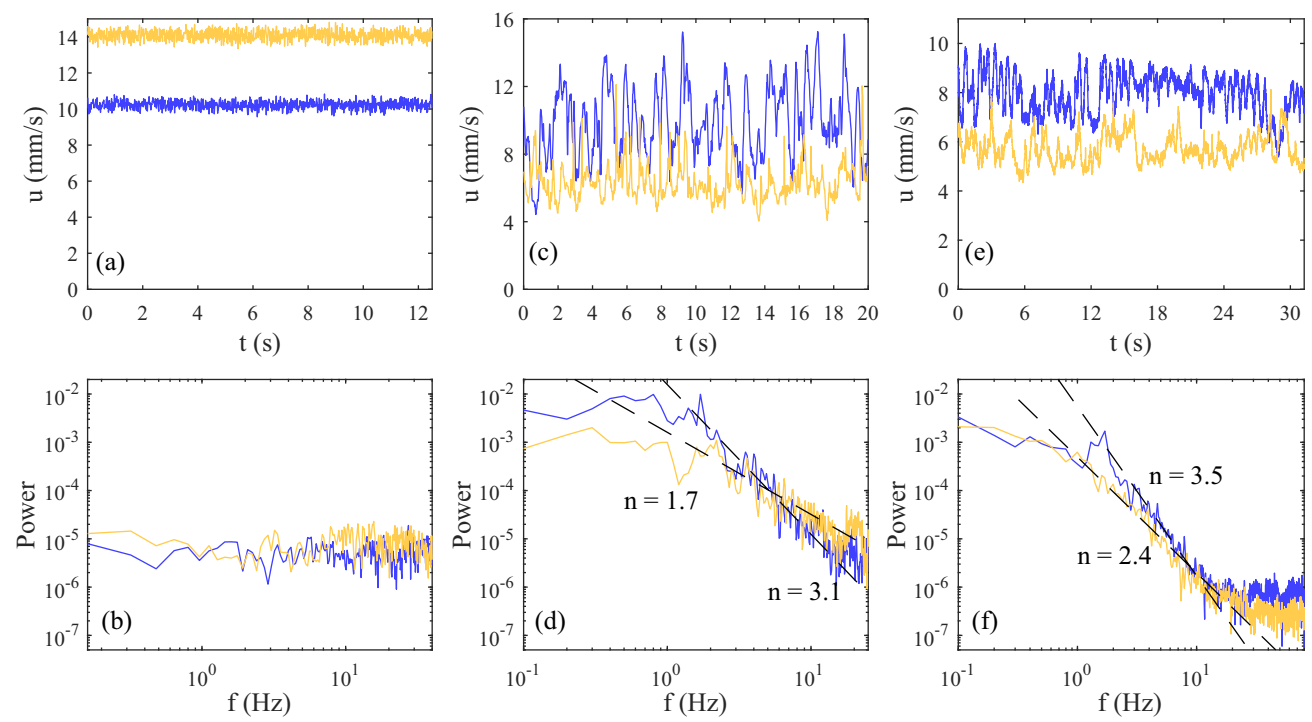

FIG. 5. Time evolution of $u$ (top) and corresponding power spectra $P$ of $u / U_{3}$ as a function of frequency $f$ (bottom) taken at $x / w_{1}=1.5$. The blue lines are for velocity measurements at $y / w_{1}=1 / 4$ and the orange lines at the centerline $y / w_{1}=0$. The dashed lines are a guide to the eye to illustrate the power law decay. (a, b) Glycerol-water mixture (90-10 weight ratio) for $\epsilon_{H}=3.69$ and $\mathrm{Re}=0.005$. (c, d) Polymer solution at $c=300$ ppm, $\mathrm{Wi}=12, \epsilon_{H}=3.69$, and $\operatorname{Re}=0.0035$. (e, f) $c=300 \mathrm{ppm}, \mathrm{Wi}=9.3, \epsilon_{H}=3.04$, and $\operatorname{Re}=0.0027$.

resolution. At the lower applied flow rates, we acquire movies at lower frequency to increase the distance traveled by the particles between two images, hence we lose some higher frequencies in the power spectrum.

Figures 5(c) and 5(e) present time series of $u$ for chaotic flows at different Wi and $\epsilon_{H}$ and the comparison with a Newtonian fluid at similar flow rate [Fig. 5(a)]. The response is different for $y=0$ and $y=w_{1} / 4$ and shows larger fluctuations at $y / w_{1}=1 / 4$. Indeed, in the two viscoelastic cases the power law exponent $n$ is higher than 3 at $y / w_{1}=1 / 4$ and $n<3$ at the centerline $y / w_{1}=0$. This is also seen in Figs. 3(n) and 3(p), where the difference of fluctuations at the centerline and at $y / w_{1}=1 / 4$ is clear.

\section{B. Decay of the time dependence}

Figure 6 shows the time evolution of $u$ at different $x$ positions in the outflow channel [see Fig. 2(a)]. The velocity time series in Figs. 6(a) and 6(b) is semiperiodic downstream of the intersection while upstream the flow is chaotic, as will be shown in Fig. 8. As seen in Fig. 6(a), close to the onset of the chaotic flow the velocity field stabilizes on a distance comparable to the width of the channel but smaller than the distance traveled by the fluid during one relaxation time, $D=Q_{3} \lambda /\left(h w_{1}\right)$. However, for higher $\epsilon_{H}$ and Wi the flow remains unstable many channel widths downstream, as seen in Fig. 6(c). Comparing Fig. 6(a) and Fig. 6(c) shows that the amplitude of the fluctuations decreases by one order of magnitude in the distance traveled during one quarter of the relaxation time for $\mathrm{Wi}=6.4$ and $\epsilon_{H}=2.08$, while for $W i=19$ and $\epsilon_{H}=3.69$ the same decay occurs only approximately over four relaxation times. The corresponding power spectra [Figs. 6(b) and 6(d)] also show a decrease of $n$ from values similar to what is expected in the elastic turbulence regime, ( $n \sim 3.7)$ [21,34] close to the intersection, to an exponent much smaller $(n \simeq 1)$ far from the intersection. Moreover, for chaotic flows, the amplitude of the velocity fluctuations increases with $\mathrm{Wi}$, as seen in Fig. 7, where we plot the standard deviation normalized by the average velocity in the chaotic regime for different Wi. The fluctuations decrease faster when Wi is smaller and eventually 

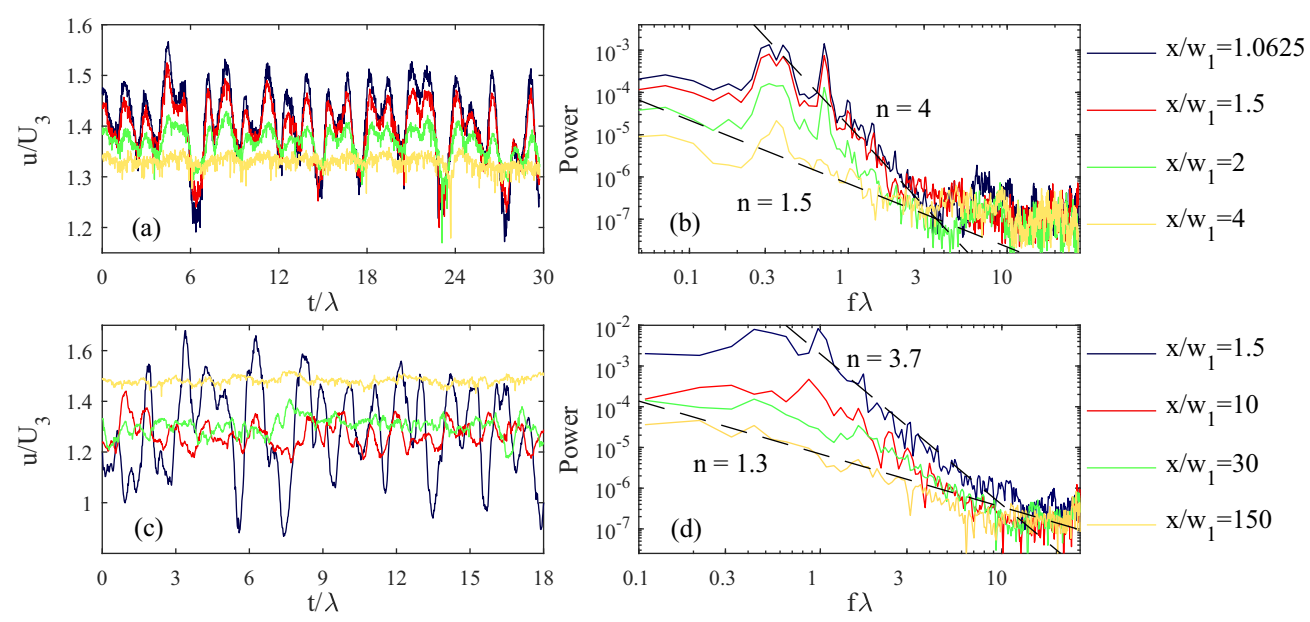

FIG. 6. (a) Normalized velocity $u / U_{3}$ measured at $y / w_{1}=1 / 4$ as a function of the normalized time $t / \lambda$ at different downstream distances: $x / w_{1}=1.0625$ (dark blue), $x / w_{1}=1.5$ (red), $x / w_{1}=2$ (light green), $x / w_{1}=4$ (very light orange), for $c=150 \mathrm{ppm}, \epsilon_{H}=2.08, \mathrm{Wi}=6.4$, and $\operatorname{Re}=0.0058$. (b) Corresponding power spectrum of $u / U_{3}$ as a function of the dimensionless frequency $\lambda f$, with the dashed lines showing the power law decay limits. (c) $u / U_{3}$ at $y / w_{1}=1 / 4$ at $x / w_{1}=1.5$ (dark blue), $x / w_{1}=10$ (red), $x / w_{1}=30$ (light green) and $x / w_{1}=150$ (very light orange), for $c=300 \mathrm{ppm}, \epsilon_{H}=3.69, \mathrm{Wi}=19$, and $\operatorname{Re}=0.0034$. (d) Corresponding power spectrum.

reach values similar to Newtonian fluids farther downstream in the outflow channel. This behavior is similar to what was observed by Pan et al. [23] for similar polymeric solutions flowing past a linear array of cylinders, where the distance required to stabilize the velocity fluctuations increases nonlinearly with the increase of Wi. Close to the onset of the elastic instability, the fluctuations of the velocity decay faster, while at high Wi the velocity fluctuations were almost independent of the distance to the perturbation [23]. In our setup, we do not achieve long-range instability, since at most the velocity fluctuations vanished at $d / w_{1} \simeq 100$. However, it may be possible to induce long-lived fluctuations for higher Wi, but this would require the use of channels with higher resistance. In our

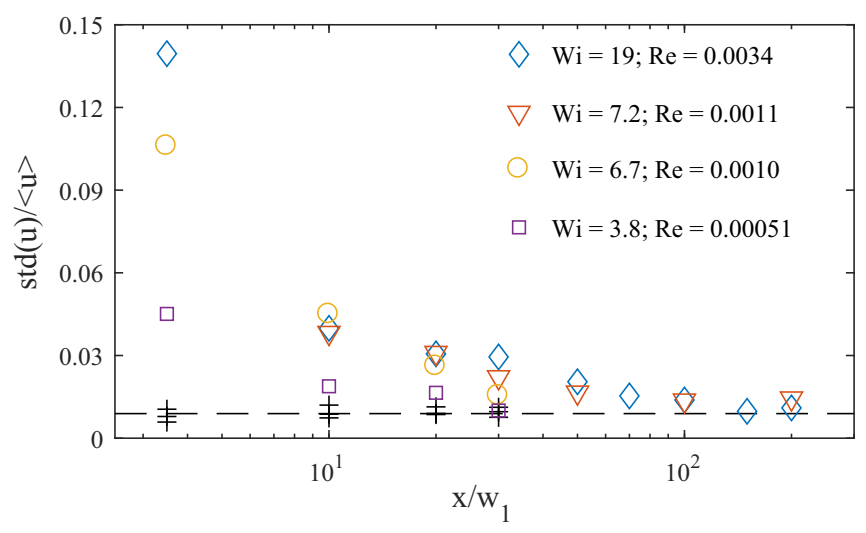

FIG. 7. Standard deviation of $u$ normalized by the time average value of $u$ for various Wi and $\epsilon_{H}=3.69$, for $c=300 \mathrm{ppm}$. The black crosses show results for a glycerol-water mixture at a 90-10 weight ratio at $\epsilon_{H}=3.69$ and $\mathrm{Re}=0.005$. The dashed line is the average value of the experiments in glycerol-water. 


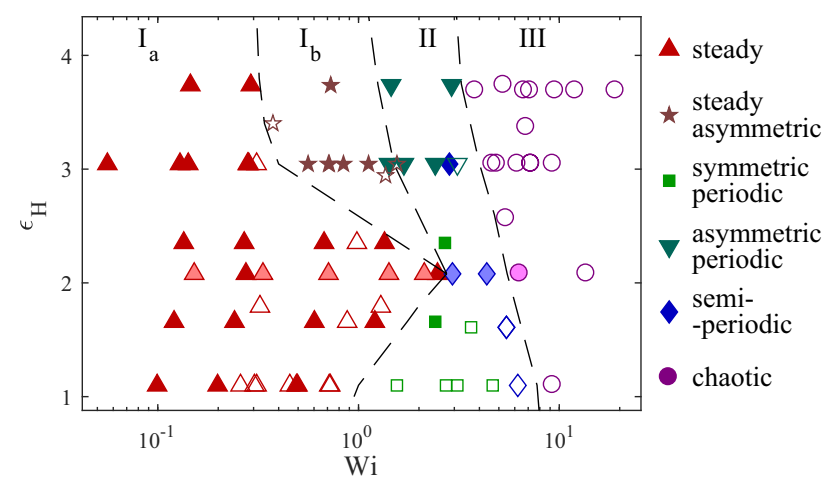

FIG. 8. Flow field classification map observed upstream of the intersection as a function of $\epsilon_{H}$ and Wi for $c=50 \mathrm{ppm}$ (full symbols), $c=150 \mathrm{ppm}$ (grayed symbols), and $c=300 \mathrm{ppm}$ (hollow symbols). The dashed lines are guides for the eyes and separate approximately the stable steady symmetric flow regime $I_{a}$, the steady asymmetric regime $\mathrm{I}_{\mathrm{b}}$, the time-dependent regime II, and the chaotic regime III.

case, we could not achieve flows for $\mathrm{Wi}>20$ because the increase in pressure resulting from the high flow rates deforms significantly the PDMS microchannels.

\section{DISCUSSION}

Figure 8 summarizes the different types of flow behavior upstream of the intersection in a $\epsilon_{H}$-Wi map. The flow field was classified as follows: for low Wi the flow is steady and symmetric [Figs. 3(a) and 3(b) and upward triangles in Fig. 8]. For higher $\epsilon_{H}$ a steady asymmetric flow is first observed increasing Wi [Figs. 3(c) and 3(d) and stars in Fig. 8] with a clear flow asymmetry in the upstream branch of the main channel. With increasing Wi, the flow becomes unsteady, either with periodic oscillations at low $\epsilon_{H}$ (squares in Fig. 8) or asymmetric and periodic at higher $\epsilon_{H}$ [Figs. 3(e) and 3(f) and downward triangles in Fig. 8]. The flow then becomes semiperiodic, with brief periodic bursts but without long time correlation, resulting in wide peaks in the power spectra followed by a strong decay with increasing frequency (diamonds in Fig. 8). Finally, at even higher Wi, the flow becomes chaotic for all $\epsilon_{H}$ tested and is characterized by large fluctuations of velocity with no defined frequency resulting in a power spectra following a power law decay with an exponent $n>3$ [Figs. 3(g) and 3(h), and circles in Fig. 8].

In all cases the flow field of the polymeric solutions changes from Newtonian-like steady and symmetric flow to chaotic flow as Wi increases, but varying $\epsilon_{H}$ leads to some notable differences. The most obvious difference is the absence of asymmetric flow for lower values of the Hencky strain. This is consistent with numerical simulations [12], where steady asymmetric flow was predicted only for $\epsilon_{H}>2.4$. We also found that the critical Wi corresponding to the onset of chaotic flow decreases with increasing $\epsilon_{H}$, showing that large extensional normal stresses generated at higher $\epsilon_{H}$ (and higher Wi) are important to the flow instability. Interestingly, for $\epsilon_{H}=2.08$, no periodic flow was observed. The main difference between our experiments and the numerical calculations of Oliveira et al. [12] is the onset of time-dependent flow. In their work, the critical Wi for time-dependent flow increases with increasing Hencky strain, while, in our experiments, this is the case at low $\epsilon_{H}$, but the time-dependent flow begins at lower Wi for $\epsilon_{H}=3.04$ than for $\epsilon_{H}=2.08$. This may be due to the differences between experiments and simulations, particularly because the numerical calculations consisted of two-dimensional (2D) flow while the experiments are realized in 3D geometries $\left(w_{1}=w_{2} / 2 \simeq h\right)$, and also because the constitutive equation used in the simulations was the upper-convected Maxwell (UCM) model, which does not include shear thinning of the viscosity as happens in the fluids used in the experiments, and predicts a much larger increase of 
first normal stress difference with shear rate. In the UCM model $N_{1} \propto \dot{\gamma}^{2}$, while in the fluids used in this work $N_{1}$ increases approximately linearly with $\dot{\gamma}$ as shown in Fig. 1(b).

Future investigations will focus on the dependency of the onset of asymmetry and time dependent flow on the channel aspect ratio, $\alpha=h / w_{1}$. This will provide a better understanding of the relevant dimensionless numbers that characterize the onset of flow instabilities. Additionally, new geometries were designed numerically to create more homogeneous, nearly constant strain rate through the intersection [35], and these ideal geometries can also provide valuable information regarding the critical conditions for the onset of flow instabilities.

\section{CONCLUSIONS}

We have shown the complex behavior of viscoelastic fluids in a flow focusing device over a wide range of Weissenberg numbers and different stretching levels measured by the Hencky strain. We have also shown that the path toward a chaotic flow depends both on Wi and $\epsilon_{H}$. For low $\epsilon_{H}$, as Wi increases, the initially stable flow becomes periodic, then semiperiodic before displaying chaotic behavior at $\mathrm{Wi} \sim 10$. For high $\epsilon_{H}$, a purely elastic instability at $\mathrm{Wi} \sim 0.3$ first renders the flow steady asymmetric, before a periodic state is obtained. Increasing Wi further leads to chaotic flow with a power law decay of the power spectra compatible with the elastic turbulence regime. The flow pattern map obtained shows that high Wi is required to reach a chaotic flow, while $\epsilon_{H}$ determines the road to instability. The flow focusing device allows to easily vary independently the level of stretching of the polymer molecules and the rate of deformation, providing a valuable platform for extensional rheology measurements. It is also useful to analyze the onset of purely elastic flow instabilities, allowing for direct comparison between numerical calculations [12] and experiments since small changes in the input parameters can greatly change the time dependency of the velocity field and the stability of the system.

\section{ACKNOWLEDGMENTS}

The research leading to these results has received funding from the European Research Council under the European Union's Seventh Framework Programme (FP/2007-2013)/ERC Grant Agreement No. 307499.

[1] P. E. Arratia, C. C. Thomas, J. Diorio, and J. P. Gollub, Elastic Instabilities of Polymer Solutions in Cross-Channel Flow, Phys. Rev. Lett. 96, 144502 (2006).

[2] S. J. Haward, T. J. Ober, M. S. N. Oliveira, M. A. Alves, and G. H. McKinley, Extensional rheology and elastic instabilities of a wormlike micellar solution in a microfluidic cross-slot device, Soft Matter 8, 536 (2012).

[3] P. C. Sousa, F. T. Pinho, M. S. N. Oliveira, and M. A. Alves, Purely elastic flow instabilities in microscale cross-slot devices, Soft Matter 11, 8856 (2015).

[4] R. J. Poole, M. A. Alves, and P. J. Oliveira, Purely Elastic Flow Asymmetries, Phys. Rev. Lett. 99, 164503 (2007).

[5] S. J. Haward, M. S. N. Oliveira, M. A. Alves, and G. H. McKinley, Optimized Cross-Slot Flow Geometry for Microfluidic Extensional Rheometry, Phys. Rev. Lett. 109, 128301 (2012).

[6] S. J. Haward, Microfluidic extensional rheometry using stagnation point flow, Biomicrofluidics 10, 043401 (2016).

[7] F. J. Galindo-Rosales, M. A. Alves, and M. S. N. Oliveira, Microdevices for extensional rheometry of low viscosity elastic liquids: A review, Microfluid Nanofluid 14, 1 (2013).

[8] A. G. Balducci, J. Tang, and P. S. Doyle, Electrophoretic stretching of DNA molecules in cross-slot nanoslit channels, Macromolecules 41, 9914 (2008). 
[9] R. Dylla-Spears, J. E. Townsend, L. Jen-Jacobson, L. L. Sohn, and S. J. Muller, Single-molecule sequence detection via microfluidic planar extensional flow at a stagnation point, Lab. Chip 10, 1543 (2010).

[10] S. Cha, T. Shin, S. S. Lee, W. Shim, G. Lee, S. J. Lee, Y. Kim, and J. M. Kim, Cell stretching measurement utilizing viscoelastic particle focusing, Anal. Chem. 84, 10471 (2012).

[11] S. L. Anna and H. C. Mayer, Microscale tipstreaming in a microfluidic flow focusing device, Phys. Fluids 18, 121512 (2006).

[12] M. S. N. Oliveira, F. T. Pinho, R. J. Poole, P. J. Oliveira, and M. A. Alves, Purely elastic flow asymmetries in flow-focusing devices, J. Non-Newtonian Fluid Mech. 160, 31 (2009).

[13] M. S. N. Oliveira, F. T. Pinho, and M. A. Alves, Extensional flow of Newtonian and Boger fluids through a flow focusing microdevice, in 3rd Micro and Nano Flows Conference Thessaloniki, Greece, 22-24 August (Brunel University, 2011).

[14] G. Juarez and P. E. Arratia, Extensional rheology of DNA suspensions in microfluidic devices, Soft Matter 7, 9444 (2011).

[15] A. Varshney, E. Afik, Y. Kaplan, and V. Steinberg, Oscillatory elastic instabilities in an extensional viscoelastic flow, Soft Matter 12, 2186 (2016).

[16] P. Pakdel and G. H. McKinley, Elastic Instability and Curved Streamlines, Phys. Rev. Lett. 77, 2459 (1996).

[17] A. N. Morozov and W. van Saarloos, An introductory essay on subcritical instabilities and the transition to turbulence in visco-elastic parallel shear flows, Phys. Rep. 447, 112 (2007).

[18] E. S. G. Shaqfeh, Purely elastic instabilities in viscometric flows, Annu. Rev. Fluid Mech. 28, 129 (1996).

[19] R. G. Larson, Instabilities in viscoelastic flows, Rheol. Acta 31, 213 (1992).

[20] A. Groisman and V. Steinberg, Elastic turbulence in a polymer solution flow, Nature (London) 405, 53 (2000).

[21] A. Groisman and V. Steinberg, Elastic turbulence in curvilinear flows of polymer solutions, New J. Phys. 6, 29 (2004).

[22] H. Y. Gan, Y. C. Lam, and N.-T. Nguyen, Polymer-based device for efficient mixing of viscoelastic fluids, Appl. Phys. Lett. 88, 224103 (2006).

[23] L. Pan, A. Morozov, C. Wagner, and P. E. Arratia, Nonlinear Elastic Instability in Channel Flows at Low Reynolds Numbers, Phys. Rev. Lett. 110, 174502 (2013).

[24] R. Grigoriev and H. G. Schuster (eds.), Transport and Mixing in Laminar Flows: From Microfluidics to Oceanic Currents (Wiley, New York, 2011).

[25] D. F. James, Boger fluids, Annu. Rev. Fluid Mech. 41, 129 (2009).

[26] Y. Jun and V. Steinberg, Elastic turbulence in a curvilinear channel flow, Phys. Rev. E 84, 056325 (2011).

[27] L. Campo-Deaño, F. J. Galindo-Rosales, F. T. Pinho, M. A. Alves, and M. S. N. Oliveira, Flow of low viscosity Boger fluids through a microfluidic hyperbolic contraction, J. Non-Newton. Fluid Mech. 166, 1286 (2011).

[28] R. B. Bird, R. C. Armstrong, and O. Hassager, Dynamics of Polymeric Liquids, Volume 1: Fluid Mechanics (Wiley, New York, 1987).

[29] P. C. Sousa, E. J. Vega, R. G. Sousa, J. M. Montanero, and M. A. Alves, Measurement of relaxation times in extensional flow of weakly viscoelastic polymer solutions, Rheol. Acta 56, 11 (2017).

[30] J. M. Dealy, Weissenberg and Deborah numbers-their definition and use, Rheology Bulletin 79, 14 (2010).

[31] R. J. Poole, The Deborah and Weissenberg numbers, Rheology Bulletin 53, 32 (2012).

[32] See Supplemental Material at http://link.aps.org/supplemental/10.1103/PhysRevFluids.2.053301 for illustrative movies of different flow regimes.

[33] L. E. Rodd, T. P. Scott, D. V. Boger, J. J. Cooper-White, and G. H. McKinley, The inertio-elastic planar entry flow of low-viscosity elastic fluids in micro-fabricated geometries, J. Non-Newtonian Fluid Mech. 129, 1 (2005).

[34] A. Fouxon and V. Lebedev, Spectra of turbulence in dilute polymer solutions, Phys. Fluids 15, 2060 (2003).

[35] F. M. P. Pimenta, Application of open-source software in the design of microfluidic devices for controlled deformation of biomolecules, Master's thesis, Faculdade de Engenharia Universidade do Porto, 2014. 